\title{
Note: Melting criterion for soft particle systems in two dimensions
}

\author{
Sergey Khrapak \\ Institut für Materialphysik im Weltraum, Deutsches Zentrum für Luft- und Raumfahrt (DLR), 82234 Weßling, \\ Germany; Aix Marseille University, CNRS, PIIM, 13397, Marseille, France; and Joint Institute \\ for High Temperatures, Russian Academy of Sciences, 125412 Moscow, Russia
}

(Received 28 February 2018; accepted 2 April 2018; published online 11 April 2018)

https://doi.org/10.1063/1.5027201

According to the Berezinskii-Kosterlitz-ThoulessHalperin-Nelson-Young (BKTHNY) theory, ${ }^{1}$ melting in two dimensions (2D) is a two-stage process. The crystal first melts by dislocation unbinding to an anisotropic hexatic fluid and then undergoes a continuous transition into an isotropic fluid. The dislocation unbinding occurs when the Young's modulus reaches the universal value of $16 \pi$,

$$
\frac{4 \mu(\mu+\lambda)}{2 \mu+\lambda} \frac{b^{2}}{k_{\mathrm{B}} T}=16 \pi,
$$

where $\mu, \lambda$ are the Lamé coefficients of the $2 \mathrm{D}$ solid, $b$ is the lattice constant, and $k_{\mathrm{B}} T$ is the thermal energy. The Lamé coefficients to be substituted in Eq. (1) should be evaluated taking into account (i) thermal softening and (ii) renormalization due to dislocation-induced softening of the crystal. ${ }^{2,3}$ Simplistic theoretical estimates using the elastic constants of an ideal crystalline lattice at $T=0$ yield melting temperatures overestimated by a factor between $\simeq 1.5$ and $\simeq 2$ for various $2 \mathrm{D}$ systems. $^{3-6}$

BKTHNY scenario has been confirmed experimentally, in particular, for systems with dipole-like interactions. ${ }^{3,7,8}$ More recently, it has been reported that 2D melting scenario depends critically on the potential softness. ${ }^{9}$ Only for sufficiently soft long-range interactions does melting proceed via the BKTHNY scenario. For steeper interactions, the harddisk melting scenario with first order hexatic-liquid transition holds. ${ }^{10-12}$

The focus of this Note is on 2D soft particle systems. It is demonstrated that a melting criterion can be introduced, which states that the melting occurs when the ratio of the transverse sound velocity of an ideal crystalline lattice to the thermal velocity reaches a certain quasi-universal value.

The Lamé coefficients of an ideal 2D lattice can be expressed in terms of the longitudinal $\left(C_{\mathrm{L}}\right)$ and transverse $\left(C_{\mathrm{T}}\right)$ sound velocities as $\mu=m \rho C_{\mathrm{T}}^{2}$ and $\lambda=m \rho\left(C_{\mathrm{L}}^{2}-2 C_{\mathrm{T}}^{2}\right)$, where $m$ and $\rho$ are the particle mass and number density. ${ }^{5,13}$ Then condition (1) can be rewritten as

$$
2 \pi \sqrt{3} v_{\mathrm{T}}^{2}=C_{\mathrm{T}}^{2}\left(1-C_{\mathrm{T}}^{2} / C_{\mathrm{L}}^{2}\right),
$$

where $v_{\mathrm{T}}=\sqrt{k_{\mathrm{B}} T / m}$ is the thermal velocity. For soft repulsive potentials, independent of space dimensionality, the following strong inequality, $C_{\mathrm{L}}^{2} / C_{\mathrm{T}}^{2} \gg 1$, holds. ${ }^{14-16}$ This implies that
Eq. (2) can be further simplified to

$$
C_{\mathrm{T}} / v_{\mathrm{T}} \simeq \text { const }
$$

at melting. The value of the constant that follows from Eq. (2) is $\simeq 3.30$. However, this does not take into account thermal and dislocation induced softening. A working hypothesis to be verified is that a simple renormalization of the constant in Eq. (3) can account for these effects. In this case, Eq. (3) would be identified as a simple 2D universal melting rule for soft particle systems.

Let us verify whether the ratio $C_{\mathrm{T}} / v_{\mathrm{T}}$ does assume a universal value at melting. We consider three exemplary 2D systems with soft long-ranged repulsive interactions: one-component plasmas with logarithmic potential (OCP $\log ),{ }^{17,19,20} 2 \mathrm{D}$ electron system with Coulomb $\propto 1 / r$ potential (OCP $1 / r),{ }^{21,22}$ and dipole-like system with $\propto 1 / r^{3}$ interaction. ${ }^{7,8,18}$ The pair-wise interaction potential $\phi(r)$ can be written in a general form as

$$
\phi(r) / k_{\mathrm{B}} T=\Gamma f(r / a),
$$

where $\Gamma$ is the coupling parameter and $a=1 / \sqrt{\pi \rho}$ is the $2 \mathrm{D}$ Wigner-Seitz radius. The system is usually referred to as strongly coupled when $\Gamma \gg 1$. The fluid-solid phase transition is characterized by a system-dependent critical coupling parameter $\Gamma_{\mathrm{m}}$ (the subscript " $\mathrm{m}$ " refers to melting). All systems considered here form hexagonal lattices in the crystalline phase (more complicated interactions and lattices should be considered separately).

The discussed soft-particle systems have been extensively studied in the literature and some relevant information is summarized in Table I. In particular, the last column lists the ratios $C_{\mathrm{T}} / v_{\mathrm{T}}$ at melting. The values presented indicate that as the potential steepness grows some weak increase of the ratio $C_{\mathrm{T}} / v_{\mathrm{T}}$ at melting is likely. At the same time, all the values are scattered in a relatively narrow range, $4.3 \pm 0.3$. This implies that Eq. (3) can be used as an approximate onephase criterion of melting of 2D crystals with soft long-ranged interactions.

As an example of the application of the proposed criterion, the melting curve of a 2D Yukawa crystal has been calculated. The Yukawa potential is characterized by $f(x)=\exp (-\kappa x) / x$, where $\kappa$ is the screening parameter (ratio of the mean interparticle separation $a$ to the screening length). This potential is used as a reasonable first approximation to describe actual interactions in colloidal suspensions and complex (dusty) 
TABLE I. Selected properties of 2D one-component plasma with logarithmic (OCP $\log$ ) and Coulomb (OCP 1/r) interactions and of the 2D system with the dipole-like interaction. Here $C_{\mathrm{T}}$ is the transverse sound velocity of an ideal triangular lattice, $v_{\mathrm{T}}$ is the thermal velocity, and $\Gamma_{\mathrm{m}}$ is the coupling parameter at melting.

\begin{tabular}{lllll}
\hline \hline System & $f(x)$ & $C_{\mathrm{T}} / v_{\mathrm{T}}{ }^{\mathrm{a}}$ & \multicolumn{1}{c}{$\Gamma_{\mathrm{m}}{ }^{\mathrm{b}}$} & $C_{\mathrm{T}} /\left.v_{\mathrm{T}}\right|_{\Gamma_{\mathrm{m}}}$ \\
\hline OCP $\log$ & $-\ln x$ & $\sqrt{\Gamma / 8}$ & $\simeq 130 \div 140$ & $\simeq 4.0 \div 4.2$ \\
OCP $1 / r$ & $1 / x$ & $0.372 \sqrt{\Gamma}$ & $\simeq 120 \div 140$ & $\simeq 4.1 \div 4.4$ \\
Dipole & $1 / x^{3}$ & $0.547 \sqrt{\Gamma}$ & $\simeq 60 \div 70$ & $\simeq 4.2 \div 4.6$ \\
\hline \hline
\end{tabular}

${ }^{a}$ See, e.g., Ref. 17 for OCP $\log$, Ref. 5 for OCP $1 / r$, and Ref. 18 for the dipole system.

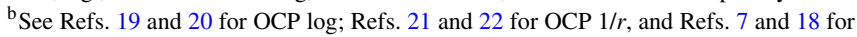
the dipole system.

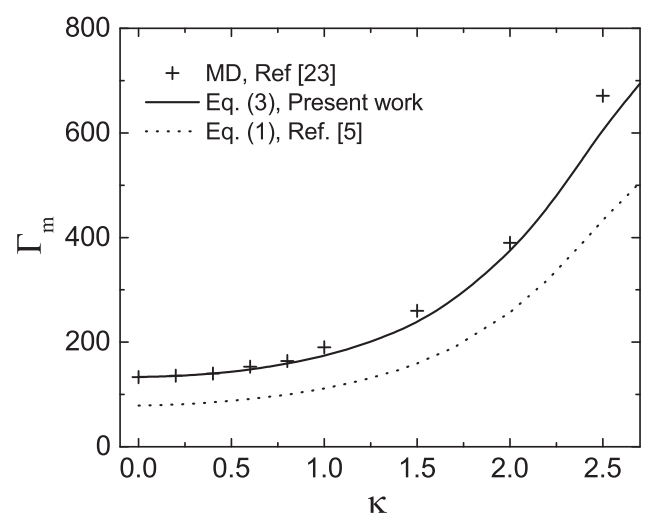

FIG. 1. Melting curve of a 2D Yukawa crystal in the $(\kappa, \Gamma)$ plane. The solid curve corresponds to the condition $C_{\mathrm{T}}=4.3 v_{\mathrm{T}}$. The symbols correspond to the results of the numerical melting "experiment." 23 The dotted line corresponds to the solution of Eq. (1) with the asymptotic $T=0$ values of elastic constants. ${ }^{5}$

plasmas. ${ }^{24-27}$ In the latter case, the screening is normally weak, ${ }^{28,29} \kappa \lesssim 1$, which corresponds to the soft interaction limit. Thermodynamics and dynamics of 2D Yukawa systems are well understood; ${ }^{23,30,31}$ simple practical expressions for thermodynamic functions $s^{32}$ and sound velocities ${ }^{16,33}$ have been derived. In particular, the transverse sound velocity of an ideal Yukawa lattice can be expressed using the Madelung constant $M(\kappa)$ as ${ }^{16}$

$$
C_{\mathrm{T}}^{2}=\frac{v_{\mathrm{T}}^{2}}{8}\left(\kappa^{2} \frac{\partial^{2} M}{\partial \kappa^{2}}+\kappa \frac{\partial M}{\partial \kappa}-M\right),
$$

where the product $M \Gamma$ defines the lattice energy per particle in units of temperature (reduced lattice sum). Using condition (3) with an "average" const $=4.3$, the dependence $\Gamma_{\mathrm{m}}(\kappa)$ for the triangular lattice has been calculated. The resulting melting line (solid curve) appears in Fig. 1. The agreement with the numerical melting "experiment" 23 is satisfactory in the weakly screened regime. An early attempt to estimate the location of the melting curve by using Eq. (1) with the asymptotic $T=0$ values of elastic constants ${ }^{5}$ is depicted by the dotted curve. A significant improvement is documented.
To conclude, a simple criterion for melting of twodimensional crystals with soft long-ranged interactions has been proposed. It states that the ratio of the transverse sound velocity of an ideal crystalline lattice to the thermal velocity is a quasi-universal number close to 4.3 at melting. Application of these criteria allows estimating melting lines in a simple yet relatively accurate manner. Two-dimensional weakly screened Yukawa systems represent just one relevant example.

Work at AMU was supported by the A*MIDEX Project (No. ANR-11-IDEX-0001-02). I thank Hubertus Thomas for reading the manuscript.

${ }^{1}$ J. M. Kosterlitz, Rev. Mod. Phys. 89, 040501 (2017).

${ }^{2}$ R. H. Morf, Phys. Rev. Lett. 43, 931 (1979).

${ }^{3}$ J. Zanghellini, P. Keim, and H. H. von Grünberg, J. Phys.: Condens. Matter 17, S3579 (2005).

${ }^{4}$ D. J. Thouless, J. Phys. C: Solid State Phys. 11, L189 (1978).

${ }^{5}$ F. M. Peeters and X. Wu, Phys. Rev. A 35, 3109 (1987).

${ }^{6}$ S. A. Khrapak and A. G. Khrapak, Contrib. Plasma Phys. 56, 270 (2016).

${ }^{7}$ K. Zahn, R. Lenke, and G. Maret, Phys. Rev. Lett. 82, 2721 (1999).

${ }^{8}$ H. H. von Grünberg, P. Keim, K. Zahn, and G. Maret, Phys. Rev. Lett. 93, 255703 (2004).

${ }^{9}$ S. C. Kapfer and W. Krauth, Phys. Rev. Lett. 114, 035702 (2015).

${ }^{10}$ E. P. Bernard and W. Krauth, Phys. Rev. Lett. 107, 155704 (2011).

${ }^{11}$ M. Engel, J. A. Anderson, S. C. Glotzer, M. Isobe, E. P. Bernard, and W. Krauth, Phys. Rev. E 87, 042134 (2013).

${ }^{12}$ A. L. Thorneywork, J. L. Abbott, D. G. Aarts, and R. P. Dullens, Phys. Rev. Lett. 118, 158001 (2017).

${ }^{13}$ L. D. Landau and E. Lifshitz, Theory of Elasticity (Elsevier, Amsterdam, 1986).

${ }^{14}$ S. A. Khrapak, Phys. Plasmas 23, 024504 (2016).

${ }^{15}$ S. Khrapak, B. Klumov, and L. Couedel, Sci. Rep. 7, 7985 (2017).

${ }^{16}$ S. Khrapak and B. Klumov, Phys. Plasmas 25, 033706 (2018).

${ }^{17}$ A. Alastuey and B. Jancovici, J. Phys. 42, 1 (1981).

${ }^{18}$ S. Khrapak, N. Kryuchkov, and S. Yurchenko, Phys. Rev. E 97, 022616 (2018).

${ }^{19}$ J. M. Caillol, D. Levesque, J. J. Weis, and J. P. Hansen, J. Stat. Phys. 28, 325 (1982).

${ }^{20}$ S. de Leeuw and J. Perram, Phys. A 113, 546 (1982).

${ }^{21}$ C. C. Grimes and G. Adams, Phys. Rev. Lett. 42, 795 (1979).

${ }^{22}$ R. C. Gann, S. Chakravarty, and G. V. Chester, Phys. Rev. B 20, 326 (1979).

${ }^{23}$ P. Hartmann, G. J. Kalman, Z. Donkó, and K. Kutasi, Phys. Rev. E 72, 026409 (2005).

${ }^{24}$ V. E. Fortov, A. G. Khrapak, S. A. Khrapak, V. I. Molotkov, and O. F. Petrov, Phys.-Usp. 47, 447 (2004).

${ }^{25}$ V. E. Fortov, A. Ivlev, S. Khrapak, A. Khrapak, and G. Morfill, Phys. Rep. 421, 1 (2005).

${ }^{26}$ A. Ivlev, H. Lowen, G. Morfill, and C. P. Royall, Complex Plasmas and Colloidal Dispersions: Particle-Resolved Studies of Classical Liquids and Solids (World Scientific, 2012).

${ }^{27}$ M. Chaudhuri, A. V. Ivlev, S. A. Khrapak, H. M. Thomas, and G. E. Morfill, Soft Matter 7, 1287 (2011).

${ }^{28}$ V. Nosenko and J. Goree, Phys. Rev. Lett. 93, 155004 (2004).

${ }^{29}$ V. Nosenko, S. K. Zhdanov, A. V. Ivlev, C. A. Knapek, and G. E. Morfill, Phys. Rev. Lett. 103, 015001 (2009).

${ }^{30}$ G. J. Kalman, P. Hartmann, Z. Donkó, and M. Rosenberg, Phys. Rev. Lett. 92, 065001 (2004).

${ }^{31}$ Z. Donko, G. J. Kalman, and P. Hartmann, J. Phys.: Condens. Matter 20, 413101 (2008).

${ }^{32}$ N. P. Kryuchkov, S. A. Khrapak, and S. O. Yurchenko, J. Chem. Phys. 146, 134702 (2017).

${ }^{33}$ I. L. Semenov, S. A. Khrapak, and H. M. Thomas, Phys. Plasmas 22, 114504 (2015). 